\title{
Design of portable near-infrared spectrum analyzer based on ARM-Linux
}

$$
\text { Saifu Deng }{ }^{\mathrm{a}} \text {, Shuangbao Wang }{ }^{* \mathrm{~b}} \text { and Linyue Wang }{ }^{\mathrm{c}}
$$

College of Optics and Electronic Information, Huazhong University of Science \& Technology, Wuhan, 430074, China

adengsaifu@126.com, bsb_wang@126.com (corresponding author), c502038062@qq.com

Keywords: near-infrared spectrum analyzer; ARM-Linux; Lock-in Amplifier; Qt interface;

Abstract. Traditional near-infrared spectrum analyzer need to be operated in assistant of PC, therefore, they are usual expensive and larger size. Here a portable near-infrared spectrum analyzer based on ARM-Linux was designed to accelerate the process of measurement automation. The measurement setup was composed of light source, optical beam splitting system, detector, data acquisition board, signal processing system, ARM controller module and corresponding embedded software system. To improve the measurement accuracy by suppressing noises and accumulating signals, the lock-in amplifier technology was applied in data processing. The characterization evaluation proved that the analyzer is ideal in practice for its intelligence, convenient and stability and repeatability.

\section{Introduction}

With the broad application in agriculture, food, pharmaceutical, petroleum and chemical industry, near-infrared spectrum (NIR) analyzer has been accepted and adopted widely by many researchers owning to its advantages in high efficiency and good resolution without any pollution or damage [1]. However, Traditional NIR analyzer has some flows like giant volume, harsh experimental conditions, and them must to be operated with PC. Therefore, using NIR analysis combined with the embedded system technology, such as a portable near-infrared spectrum analyzer based on ARM-Linux was designed in this work. The test can be executed without the assistance of PC, and high stability and accuracy is achieved

\section{System Framework}

The design purpose is to synthesize a near-infrared monochromator and embedded hardware and software to develop a portable and intelligent near-infrared spectrum analyzer. The schematic diagram of the whole system is displayed in figure 1 . It is divided into mainly 3 parts. The three parts includes light source and beam splitting system, data acquisition and processing system and ARM-Linux controller module [2].

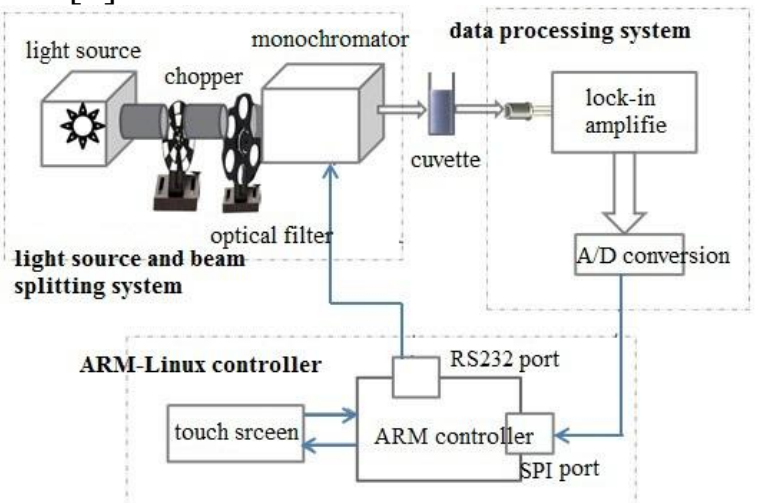

Fig. 1. Schematics of system framework.

When the light source emits broadband beam, beam splitting system disperses it into monochromatic lights ranged by wavelength. The monochromatic lights penetrate through sample and detected by photoelectric detector that transferring the light information into electrical signal. Then, the intensity of near-infrared spectrum can be drawn in screen. According to Beer-Lambert 
Law, the structure and composition of the certain molecule group of the sample can be calculated. Therefore, qualitative and quantitative analysis can be achieved.

\section{Hardware System Design}

\section{Light Source and Beam Splitting System}

The light source and beam splitting system in figure 1 includes light source v chopper and monochromator. In this work, a $30 \mathrm{~W}$ tungsten halogen lamp was used due to its characteristic energy distribution at the wavelength band of near-infrared, which changes from $300 \mathrm{~nm}$ to $2500 \mathrm{~nm}$. To eliminate the interference of noises and temperature drifting, optical chopper was applied to modulate the $\mathrm{DC}$ signal into $\mathrm{AC}$ signal which frequency is set to $10 \mathrm{~Hz}$. Using grating scanning monochromator, the entire spectrum can be detected at the exit slit.

Data Acquisition and Processing System

The data acquisition and processing system are mainly consisted of photoelectric conversion module, lock-in amplifier circuit and analog-to-digital (AD) conversion module. They convert light signal into electronic signal, and then filter and amplifier to acquire analog voltage signal, and finally collected by ARM processor.

Photoelectric Conversion Module

To obtain the intensity of light, G21-3-66 PbSe infrared detector produced by CalSensors Inc. was applied to convert light signal into electronic signal. G21-3-66 PbSe infrared detector has a wide response spectrum from 1.0 to $5.5 \mu \mathrm{m}$ and high SNR (Signal to Noise Ratio) up to 500 [4], along with high sensitivity and response speed. The schematic diagram of detector is shown in fig. 2 while Vbias represents bias voltage and RL is load. When the light is incident on the photosensitive material of detector, the voltage of two ports would change, so they will output voltage.

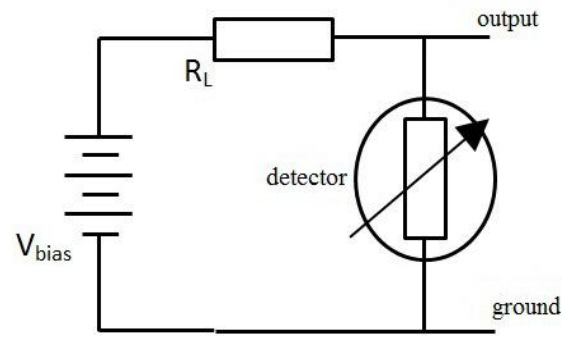

Fig. 2. Schematic diagram of G21-3-66 PbSe infrared detector

\section{Lock-in Amplifier Circuit}

The converted electronic signals have a low SNR and weak noise resistance so that they are unable to be detected in normal methods. Therefore, lock-in amplifier are applied to extract weak signals from noises. Lock-in amplifier is actually a synchronous relevance detector based on the theory of cross-correlation. It can extract the signal from massive non-relevant noises on the basis of cross-correlation between signal and reference which has the same frequency and phase. The schematic diagram is described as fig. 3 .

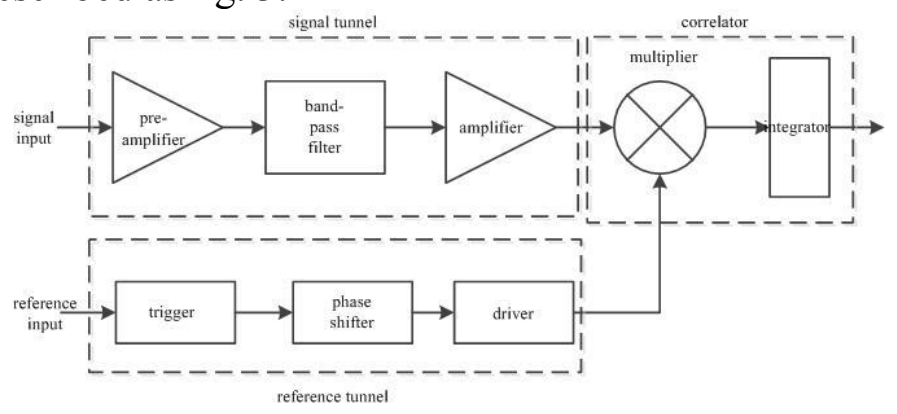

Fig. 3. Schematic diagram of lock-in amplifier circuit.

From the figure above we can see that the circuit of lock-in amplifier are mainly consisted of signal tunnel, reference tunnel and correlator. The details are shown as fig. 4 . 


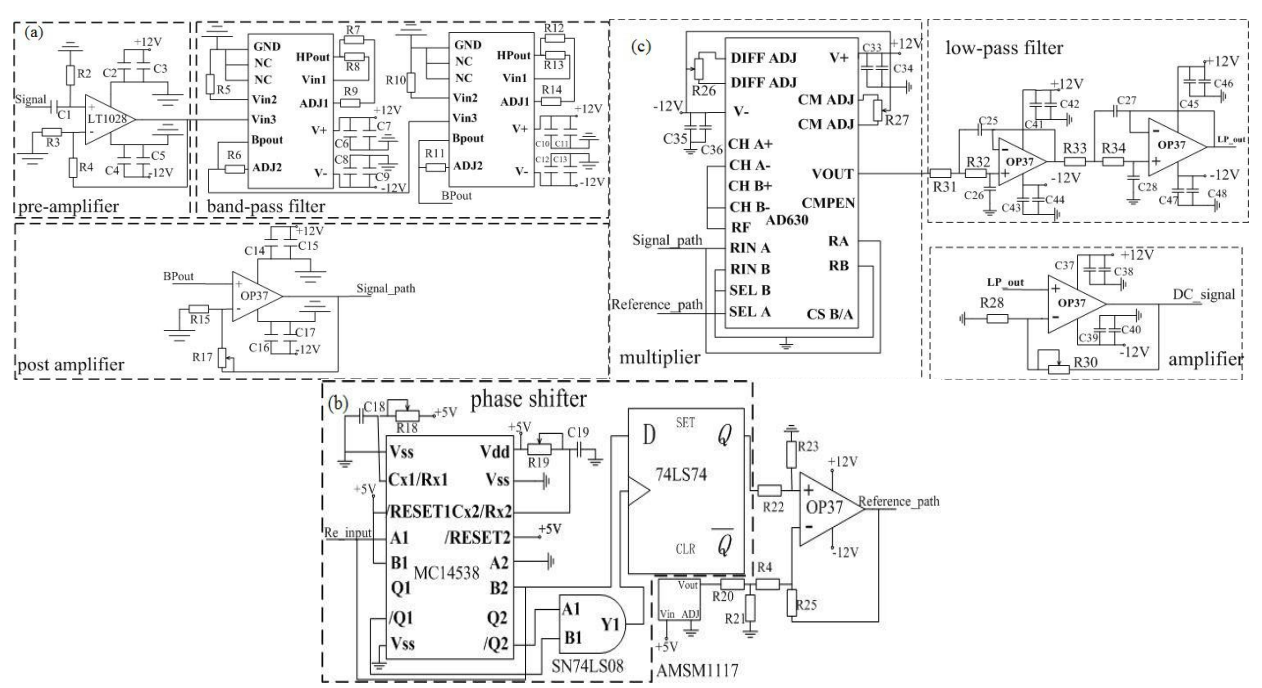

Fig. 4. Circuit diagram of (a) signal tunnel (b) reference tunnel and (c) correlator in lock-in amplifier.

Through signal tunnel, the noise in the test signal could be effectively restrained and the test signal will be sent to the multiplier in the correlator. The main signal processing of reference tunnel includes signal amplification, shaping and phase-shifting. Processed reference signal will also be sent to the multiplier. The corrector is the core component of lock-in amplifier, which does phase-sensitive detection of test signal on the basis of reference signal, and output low noise signal.

The final output of lock-in amplifier circuit is an analog direct current voltage. 12-bit A/D conversion TLC 2543 by TI convert analog signal to digital signal and then processed by ARM processor.

\section{Software System Design}

The targets of analyzer are located in intelligent operation and automatically completing the task of controlling, data acquisition and storage, drawing and processing. To achieve the goals, Linux system [7] on ARM was cut and transplanted because it can support multitasking and multithreading. The software system design of near-infrared spectrum analyzer is mostly consisted of the driver program of Linux system and Qt Embedded human-computer interface.

\section{Qt Embedded}

Qt [8] is an application framework with $\mathrm{C}++$ graphical user interface developed by TrollTech company, and Qt Embedded is the version for embedded system. Qt Embedded has been widely applied on many kinds of portable instrument for its remarkable cross-platform compatibility, abundant port and convenient operation. In this work, the parameters initiation, spectrum detection and data acquisition and processing are all completed by Qt, and each module is organized as fig. 5.

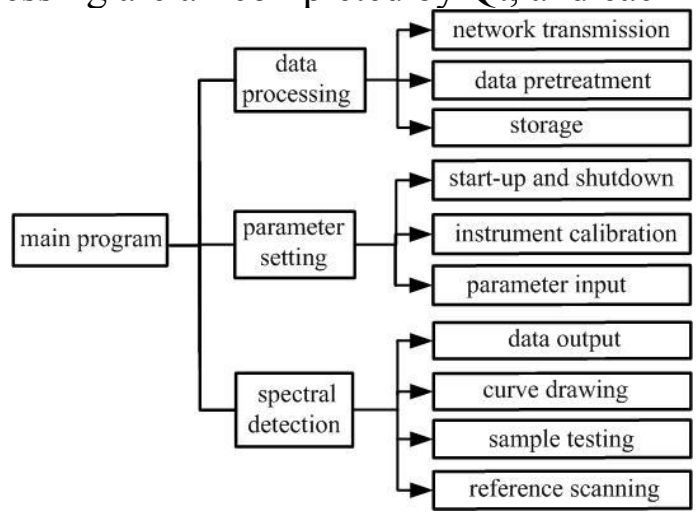

Fig. 5. The organized pattern of each module in Qt.

Acquisition Program

When software system started, the initial wavelength and scanning step must be set to the right value at the very beginning. After all the parameter configuration was completed, the system will scan 
the spectrum of the background and sample from the start. When scanning was accomplished, the software system will calculate the transmission and absorbance of the sample within the set band and draw curves at LCD screen in real-time. At the same time, the data collected can be transmit to the host PC via local area network or USB flash drive. The Qt graphical user interface are shown as fig. 6.

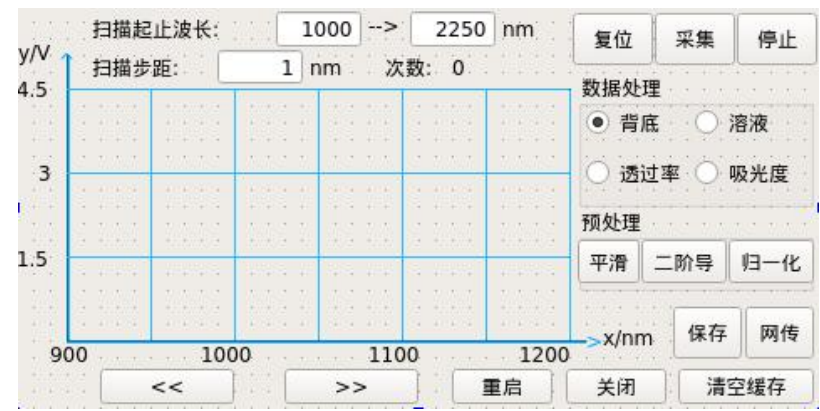

Fig. 6. Graphical user interface.

In the process of sample measurement, the transmission spectrum of reference sample was measured as background at the very beginning. Then the sample was tested to calculate the absorbance curve in near-infrared wavelength band.

\section{Results}

Accuracy

To testify system's accuracy, VERTEX70 Fourier transform infrared spectrometer by Bruker was used as reference. The absorbance curve of perspex was scanned and the result are shown as following.
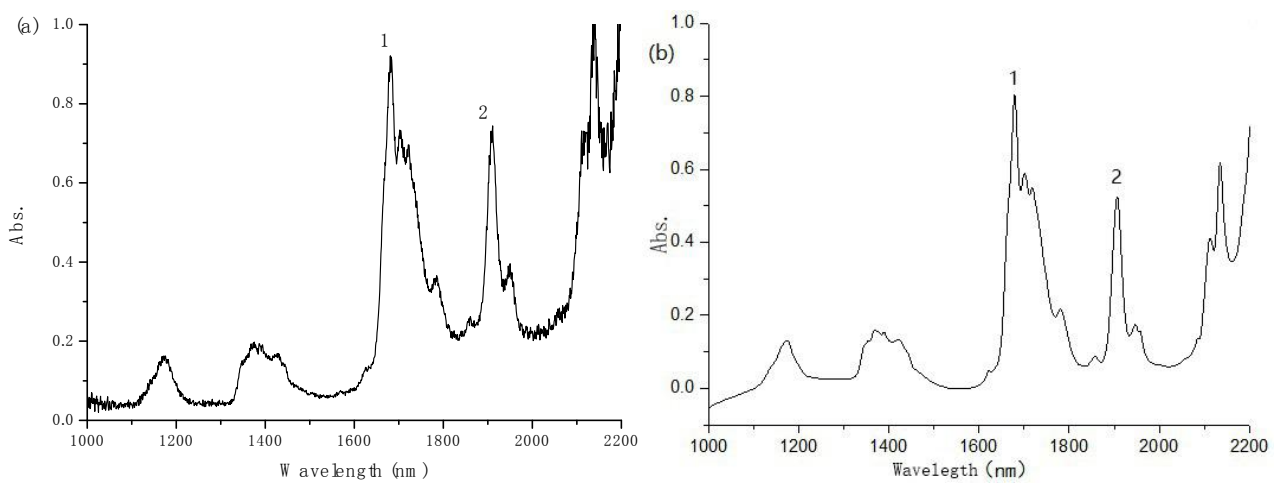

Fig. 7. The absorbance curve of Perspex (a) measured and (b)standard

Table 1. Comparison of measured peak position and standard

\begin{tabular}{ccc}
\hline Peak position & Measured & Standard \\
\hline 1 & $1682 \mathrm{~nm}$ & $1679 \mathrm{~nm}$ \\
2 & $1911 \mathrm{~nm}$ & $1908 \mathrm{~nm}$ \\
\hline
\end{tabular}

The results in Figs. 7 show an excellent agreement between measured and standard. The peak position is fairly coincident despite of some burr at the bottom of curves. From the table 1 we can see that the systematic deviation of peak position of absorbance is about $3 \mathrm{~nm}$, which verified the NIR analyzer accuracy.

\section{Repeatability}

The wavelength repeatability can be revealed by the standard deviation of peak wavelength after the multiple measurements of the same sample. Turning on the analyzer, and waiting for 5 minutes in order to gain stable data. The absorbance curve of isooctane was tested ten times continuously, and the absorption wavelength peak was extracted as followings. 
Table 2. Measured peak position of isooctane's absorption wavelength

\begin{tabular}{cc|cc}
\hline times & Peak position $[\mathrm{nm}]$ & Times & Peak position $[\mathrm{nm}]$ \\
\hline 1 & 1195 & 6 & 1194 \\
2 & 1194 & 7 & 1195 \\
3 & 1195 & 8 & 1195 \\
4 & 1194 & 9 & 1195 \\
5 & 1195 & 10 & 1195 \\
\hline \multicolumn{4}{l}{} \\
\hline
\end{tabular}

The deviation of peak wavelength in ten times is 0.483 by calculation. As a result, the wavelength repeatability is within $1 \mathrm{~nm}$.

\section{Conclusions}

A portable near-infrared spectrum analyzer based on ARM and embedded Linux system was designed on the rely of near-infrared spectrum technology. Crossing-structure of an asymmetric Czerny-Turner beam splitting system was applied due to its compact structure and it can also efficiently weaken stray light. Lock-in Amplifier Circuit had a remarkable impact on improving SNR in signal processing. The whole process of testing and analyzing can be operated by touch screen in Qt graph human-computer interface. The near-infrared spectrum analyzer we designed has achieved automatic spectrum scanning, data processing, and the transmission and absorbance calculation, with the advantages of highly intelligence and easy maneuverability. The stability and accuracy is also up to standard, providing a certain basis for the development of spectrum instrument.

\section{Acknowledgements}

This work was financially supported by the Fundamental Research Funds for the Central Universities (HUST-2015049), and also partly supported by the creative fund of CETC NO.46 Research Institute.

\section{References}

[1] X.C. Zhang, J.Z. Wu, Y. Zhang: Near-infrared Spectrum Analysis Technology and Application in Modern Agriculture (Publishing House of Electronics Industry, Beijing 2012).

[2] J. Zhao, X.J. Ye, Y.L. Wu, X.Q. Lian, Z.G. Duan: Instrument Technique and Sensor Vol. 3 (2014), p.26

[3] Y. An, Q. Sun, Y. Liu, C. Li: Chinese Optics and Applied Optics Abstracts Vol. 5 (2012), p. 470

[4] N.E.P. G21-3-66 Detector Test Report (2011)

[5] Y. Zhang, J.L. Zhang, X.Y Du: Piezoelectrics \& Acoustooptics Vol. 33 (2011), p. 674

[6] Z.A. Jia, P.P. Wang, X.G. Xiao, H. Zhou, W. Wang: Instrument Technique and Sensor Vol. 4 (2012), p. 104

[7] W.F. Liu, C.Y. Li, S.P. Li: Journal of Zhejiang University (Engineering Science) Vol. 38 (2004), p. 447

[8] J. Blanchette, M. Summerfield: C++ GUI Programming with Qt 4 (Trolltech Press, 2008) 